\title{
BOUNDS FOR THE DIAMETER OF THE WEIGHT POLYTOPE
}

\author{
SASCHA KURZ
}

\begin{abstract}
A weighted game or a threshold function in general admits different weighted representations even if the sum of non-negative weights is fixed to one. Here we study bounds for the diameter of the corresponding weight polytope. It turns out that the diameter can be upper bounded in terms of the maximum weight and the quota or threshold. We apply those results to approximation results between power distributions, given by power indices, and weights.
\end{abstract}

\section{INTRODUCTION.}

Consider a stock corporation whose shares are hold by three major stockholders owning $35 \%, 34 \%$, and $17 \%$, respectively. The remaining $14 \%$ are widely spread. Assuming that decisions are made by a simple majority rule, all three major stockholders have equal influence on the company's decisions, while the private shareholders have no say. To be more precise, any two major stockholders can adopt a proposal, while the private shareholders together with an arbitrary major stockholder need further affirmation. Such decision environments can be captured by means of weighted voting games. Formally, a weighted (voting) game consists of a set of players or voters $N=\{1, \ldots, n\}$, a vector of non-negative weights $w=\left(w_{1}, \ldots, w_{n}\right)$, and a positive quota $q$. A proposal is accepted if and only if the weight sum of its supporters meets or exceeds the quota.

Committees that decide between two alternatives have received wide attention. Von Neumann and Morgenstern introduced the notion of simple games, which is a super class of weighted games, in [20]. Examples of decision-making bodies that can be modeled as weighted games are the US Electoral College, the Council of the European Union, the UN Security Council, the International Monetary Fund or the Governing Council of the European Central Bank. Many applications seek to evaluate players' influence or power in simple or weighted games, see, e.g., [13]. The initial example illustrates that shares or weights can be a poor proxy for the distribution of power. Using the taxicab metric, i.e., the $\|\cdot\|_{1}$-distance, the corresponding distance between shares and relative power is $\left|0.35-\frac{1}{3}\right|+\left|0.34-\frac{1}{3}\right|+\left|0.17-\frac{1}{3}\right|+|0.14-0| \approx 32.67 \%$. If the weights add up to one, then we speak of relative or normalized weights. The insight that the power distribution differs from relative weights, triggered the invention of socalled power indices like the Shapley-Shubik index [18], the Penrose-Banzhaf index [2], or the nucleolus [17]. Due to the combinatorial nature of most of those power indices, qualitative assessments are technically demanding and large numbers of involved parties cause computational challenges [3]. 
One reason for the difference between relative weights and power is that a weighted game permits different representations. If there are two normalized representations whose weight vectors are at large distance than at least one of the relative weight vectors also has a large distance to the power distribution. So, here we study bounds for the diameter of the weight polytope, i.e., bounds for the maximum distance between two normalized vectors of the same weighted game. We will study those bounds in terms of the number of players, the relative quota, and the maximum relative weight in a given representation of the game.

Each weighted game, also called threshold function, admits a representation with integer weights. Bounds for the necessary magnitude of integer weighted are studied in the literature, see e.g. [1] and the references therein.

The remaining part of the paper is structure as follows. In Section 2 we give the necessary definitions for simple games, weighted games and the weight polytope. Upper bounds on the diameter of the weight polytope are given in Section 4 and worst case lower bounds are given in Section 3. Applications to approximation results for power indices are given in Section 5 before we draw a brief conclusion in Section 6 . Some lengthy or more technical proofs are moved to an appendix.

\section{The Weight POLYTope of A Weighted GAME.}

For a positive integer $n$ let $N=\{1, \ldots, n\}$ be the set of players. A simple game is a mapping $v: 2^{N} \rightarrow\{0,1\}$ from the subsets of $N$ to binary outcomes satisfying $v(\emptyset)=0, v(N)=1$, and $v(S) \leq v(T)$ for all $\emptyset \subseteq S \subseteq T \subseteq N$. The interpretation in the context of binary voting systems is as follows. A subset $S \subseteq N$ is considered as the set of players that are in favor of a proposal, i.e., which vote "yes". If $v(S)=1$ we call coalition $S$ winning and losing otherwise. By $\mathcal{W}(v)$ we denote the set of winning coalitions and by $\mathcal{L}(v)$ we denote the set of losing coalitions of $v$. If coalition $S$ is winning but each proper subset is losing, then we call $S$ minimal winning. Similarly, if $S$ is losing but each proper superset of $S$ is winning, then we call $S$ maximal losing. By $\mathcal{W}^{m}(v)$ we denote the set of minimal winning and by $\mathcal{L}^{m}(v)$ we denote the set of maximal losing coalitions. $v(S)$ encodes the group decision, i.e., $v(S)=1$ if the proposal is accepted and $v(S)=0$ otherwise. So, these assumptions for a simple game are quite natural for a voting system with binary options in the input and output domain. The dual $v^{d}$ of a simple game $v$ is defined via $v^{d}(S)=v(N)-v(N \backslash S)=1-v(N \backslash S)$ and is a simple game itself. If $v(S)=v(S \cup\{i\})$ for all $S \subseteq N$, then we call player $i$ a null player. Player $i$ is a passer if $v(\{i\})=1$. Two players $i$ and $j$ are equivalent if $v(S \cup\{i\})=v(S \cup\{j\})$ for all $S \subseteq N \backslash\{i, j\}$.

A simple game $v$ is called weighted if there exist weights $w \in \mathbb{R}_{\geq 0}^{n}$ and a quota $q \in \mathbb{R}_{>0}$ such that $v(S)=1$ if and only if $w(S):=\sum_{i \in S} w_{i} \geq q$. From the conditions of a simple game we conclude $0<q \leq w(N)$. If $w(N)=1$ we speak of normalized or relative weights, where $0<q \leq 1$. We denote the respective game by $v=[q ; w]$ and refer to the pair $(q ; w)$ as a weighted representation, i.e., we can have $[q ; w]=\left[q^{\prime} ; w^{\prime}\right]$ but $(q ; w) \neq\left(q^{\prime} ; w^{\prime}\right)$. The example from the introduction can, e.g., be represented by 
$(51 \% ; 35 \%, 34 \%, 17 \%, 14 \%),\left(\frac{1}{2} ; \frac{1}{3}, \frac{1}{3}, \frac{1}{3}, 0\right)$, or $(6 ; 4,3,3,1)$, where the fourth player mimics the private shareholders.

Lemma 2.1. If $(q ; w)$ is a normalized representation of a weighted game $v$, then (1$q+\varepsilon ; w)$ is a normalized representation of the dual game $v^{d}$ for each $0<\varepsilon<\min \{q-$ $w(S) \mid S \in \mathcal{L}(v)\}$.

Proof. Proof. For each losing coalition $S$ of $v^{d}$ the coalition $N \backslash S$ is winning in $v$, so that $w(N \backslash S)=1-w(S) \geq q$ and $w(S) \leq 1-q<1-q+\varepsilon$. Now let $S$ be a winning coalition, so that $N \backslash S$ is losing in $v$ and $\varepsilon<q-w(N \backslash S)=q-1+w(S)$, which is equivalent to $w(S)>1-q+\varepsilon$. Since $\emptyset$ is a losing coalition in $v$ we have $\varepsilon<q-w(\emptyset)=q$, so that $1-q+\varepsilon<1$.

Note that $\min \{q-w(S) \mid S \in \mathcal{L}(v)\}>0$.

Given a weighted game $v$ and a weight vector $w$ we can find a quota $q \in \mathbb{R}_{>0}$ such that $v=[q ; w]$ if and only if the largest weight of a losing coalition is strictly smaller than the smallest weight of a winning coalition. Thus, see e.g. [9, Lemma 3.2], the set of feasible normalized weight vectors is given by

$$
\begin{aligned}
& \left\{w \in \mathbb{R}_{\geq 0}^{n} \mid w(N)=1, v(S)>v(T) \quad \forall S \in \mathcal{W}(v), T \in \mathcal{L}(v)\right\} \\
= & \left\{w \in \mathbb{R}_{\geq 0}^{n} \mid w(N)=1, v(S)>v(T) \quad \forall S \in \mathcal{W}^{m}(v), T \in \mathcal{L}^{m}(v)\right\} .
\end{aligned}
$$

Definition 2.2. For a weighted game $v$ we define the weight polytope of $v$ by

$$
\mathbf{W}(v)=\left\{w \in \mathbb{R}_{\geq 0}^{n} \mid w(N)=1, v(S) \geq v(T) \quad \forall S \in \mathcal{W}^{m}(v), T \in \mathcal{L}^{m}(v)\right\}
$$

and call

its diameter.

$$
\operatorname{diam}(\mathbf{W}(v))=\max \left\{\left\|w-w^{\prime}\right\|_{1} \mid w, w^{\prime} \in \mathbf{W}(v)\right\}
$$

While not any element in $\mathbf{W}(v)$ can be completed by a suitable quota $q \in(0,1]$ to a normalized representation $(q ; w)$, Definition 2.2 makes sense nevertheless since $\operatorname{dim}(\mathbf{W}(v))=n-1$, see e.g. [9. Lemma 3.4]. More concretely, for each weighted game $v$ and each $\varepsilon \in \mathbb{R}_{>0}$ there are $w, w^{\prime} \in \mathbf{W}(v)$ and $q, q^{\prime} \in(0,1]$ such that $v=[q ; w]=$ $\left[q^{\prime} ; w^{\prime}\right]$ and

$$
\operatorname{diam}(\mathbf{W}(v))-\varepsilon \leq\left\|w-w^{\prime}\right\|_{1} \leq \operatorname{diam}(\mathbf{W}(v)) .
$$

Given the indicated linear programming formulation, diam $(\mathrm{W}(v))$ can be computed in polynomial time (depending on the number of minimal winning and maximal losing coalitions). The same is true if we replace $\|\cdot\|_{1}$ by the maximum norm $\|x\|_{\infty}=$ $\max \left\{x_{i} \mid 1 \leq i \leq n\right\}$ for $x \in \mathbb{R}^{n}$. We denote the corresponding diameter by $\operatorname{diam}^{\infty}(\mathrm{W}(v))$. For an arbitrary $p$-norm $\|\cdot\|_{p}$ with $1<p<\infty$, we can obtain lower and upper bounds via $\|x\|_{\infty} \leq\|x\|_{p} \leq\|x\|_{1}$, so that we restrict ourselves to these two distance functions. The bound $\|x\|_{\infty} \leq\|x\|_{1}$ can be slightly improved in our context.

Lemma 2.3. For $w, w^{\prime} \in \mathbb{R}_{\geq 0}^{n}$ with $\|w\|_{1}=\left\|w^{\prime}\right\|_{1}=1$, we have $\left\|w-w^{\prime}\right\|_{\infty} \leq$ $\frac{1}{2}\left\|w-w^{\prime}\right\|_{1}$. 
Proof. Proof. With $S:=\left\{1 \leq i \leq n \mid w_{i} \leq w_{i}^{\prime}\right\}$ and $A:=\sum_{i \in S}\left(w_{i}^{\prime}-w_{i}\right), B:=$ $\sum_{i \in N \backslash S}\left(w_{i}-w_{i}^{\prime}\right)$, where $N=\{1, \ldots, n\}$, we have $A-B=0$ since $\|w\|_{1}=\left\|w^{\prime}\right\|_{1}$ and $w, w^{\prime} \in \mathbb{R}_{\geq 0}^{n}$. Thus, $\left\|w-w^{\prime}\right\|_{1}=2 A$ and $\left\|w-w^{\prime}\right\|_{\infty} \leq \max \{A, B\}=A$.

What can be said about $\operatorname{diam}(\mathrm{W}(v))$ and $\operatorname{diam}^{\infty}(\mathrm{W}(v))$ in general without solving the specific linear programs? Obviously, we have $\operatorname{diam}(\mathrm{W}(v)) \leq 2$ and $\operatorname{diam}^{\infty}(\mathrm{W}(v)) \leq$ 1. These bounds are asymptotically attained for $n \geq 2$ and $v=[n ;(1, \ldots, 1)]$, i.e., for any $0<\varepsilon<\frac{1}{n}$ we can set $w=(1-(n-1) \cdot \varepsilon, \ldots, \varepsilon)$, w $w^{\prime}=(\varepsilon, \ldots, \varepsilon, 1-(n-1) \cdot \varepsilon)$, $q=q^{\prime}=1-\varepsilon$ so that $v=[q ; w]=\left[q^{\prime} ; w^{\prime}\right],\left\|w-w^{\prime}\right\|_{1}=2 \cdot(1-n \varepsilon)$, and $\left\|w-w^{\prime}\right\|_{\infty}=1-2 \varepsilon$. In other words, $(1,0, \ldots, 0),(0, \ldots, 0,1) \in \mathbf{W}([n ; 1, \ldots, 1])$ attain the desired distances. For the weighted game $v$ with $n=1$ players we have $\operatorname{diam}(\mathbf{W}(v))=\operatorname{diam}^{\infty}(\mathbf{W}(v))=0$ since $\mathbf{W}(v)=\{(1)\}$.

In order to obtain tighter bounds for the diameter of the weight polytope we need more information besides the number of players. Given an exemplary normalized representation $(q ; w)$, we study key parameters like the relative quota $q \in(0,1]$ or the maximum relative weight $\Delta(w):=\|w\|_{\infty} \in(0,1]$, where we write $\Delta$ whenever $w$ is clear from the context. Besides this, also more sophisticated invariants of weight vectors have been studied in applications. The so-called Laakso-Taagepera index a.k.a. Herfindahl-Hirschman index, c.f. [12], is used in Industrial Organization to measure the concentration of firms in a market, see, e.g., [4], and given by

$$
L(w)=\left(\sum_{i=1}^{n} w_{i}\right)^{2} / \sum_{i=1}^{n} w_{i}^{2} .
$$

for $w \in \mathbb{R}_{\geq 0}^{n}$ with $w \neq 0$. In general we have $1 \leq L(w) \leq n$. If the weight vector $w$ is normalized, then the formula simplifies to $L(w)=1 / \sum_{i=1}^{n} w_{i}^{2}$. Under the name "effective number of parties" the index is widely used in political science to measure party fragmentation, see, e.g., [11]. However, we observe the following relations between the maximum relative weight $\Delta=\Delta(w)$ and the Laakso-Taagepera index $L(w)$ :

Lemma 2.4. For $w \in \mathbb{R}_{\geq 0}^{n}$ with $\|w\|_{1}=1$, we have

$$
\frac{1}{\Delta} \leq \frac{1}{\Delta(1-\alpha(1-\alpha) \Delta)} \leq L(w) \leq \frac{1}{\Delta^{2}+\frac{(1-\Delta)^{2}}{n-1}} \leq \frac{1}{\Delta^{2}}
$$

for $n \geq 2$, where $\alpha:=\frac{1}{\Delta}-\left\lfloor\frac{1}{\Delta}\right\rfloor \in[0,1)$. If $n=1$, then $\Delta=L(w)=1$.

Proof. Proof. Optimize $\sum_{i=1}^{n} w_{i}^{2}$ with respect to the constraints $w \in \mathbb{R}^{n},\|w\|_{1}=1$, and $\Delta(w)=\Delta$, see the appendix for the technical details.

So, any lower or upper bound involving $L(w)$ can be replaced by a bound involving $\Delta$ instead. Since $\Delta$ has nicer analytical properties and requires less information on $w$, we stick to $\Delta$ in the following. We remark that there are similar inequalities for other indices measuring market concentration. Upper bounds on $\operatorname{diam}(\mathrm{W}(v))$, in terms of $n$, 
$q$, and $\Delta$, will be given in Section 4 and worst case lower bounds for $\operatorname{diam}(\mathrm{W}(v))$ and $\operatorname{diam}^{\infty}(\mathbf{W}(v))$ will be given in Section 3 .

\section{WORST CASE LOWER BOUNDS FOR THE DIAMETER OF THE WEIGHT POLYTOPE.}

For integers $1 \leq k \leq s$ and $t \geq 0$ we denote by $v_{k, s, t}$ the weighted game with $s$ players of weight one, $t$ players of weight zero, and a quota of $k$, i.e., $v_{k, s, t}=$ $[k ; 1, \ldots, 1,0, \ldots 0]$. Players $1, \ldots, s$ are pairwise equivalent as well as players $s+$ $1, \ldots s+t$, which are null players. If $k=1$, then each player $1 \leq i \leq s$ is a passer. First we study lower bounds for the diameter of those weighted games.

Lemma 3.1. For integers $1 \leq k<s$ and $t \geq 0$ we have

$$
\operatorname{diam}\left(\mathbf{W}\left(v_{k, s, t}\right)\right) \geq \max \left\{\frac{1}{10 k}, \frac{1}{10(s-k)}\right\} \quad \text { and } \operatorname{diam}^{\infty}\left(\mathbf{W}\left(v_{k, s, t}\right)\right) \geq \frac{1}{s} .
$$

Proof. Proof. Let $S=\{1, \ldots s\}$ and $T=\{s+1, \ldots, s+t\}$. We start with the lower bound for $\operatorname{diam}\left(\mathrm{W}\left(v_{k, s, t}\right)\right)$. If $s \equiv 0(\bmod 2)$ then we set $S_{1}=\{1, \ldots, s / 2\}, S_{0}=\emptyset$, and $S_{-1}=\{s / 2+1, \ldots, s\}$. If $s \equiv 1(\bmod 2)$, then we set $S_{1}=\{1, \ldots,(s-1) / 2\}$, $S_{0}=\{(s+1) / 2\}$, and $S_{-1}=\{(s+3) / 2, \ldots, s\}$. Let $0 \leq \gamma \leq \frac{1}{s}$ be a parameter that we specify latter depending on further case differentiations. With this, we set $w_{i}=\frac{1}{s}+\gamma$ for all $i \in S_{1}, w_{i}=\frac{1}{s}$ for all $i \in S_{0}, w_{i}=\frac{1}{s}-\gamma$ for all $i \in S_{-1}, w_{i}=\bar{w}_{i}=0$ for all $i \in T$, and $\bar{w}_{i}=w_{s+1-i}$ for all $i \in S$. It is easily verified that $w \in \mathbb{R}_{\geq 0}^{s+t}$ and $\|w\|_{1}=1$. In order to conclude $w \in \mathbf{W}\left(v_{k, s, t}\right)$ it suffices to check $w(U)+w(T)=w(U) \leq w(V)$ for all $U, V \subseteq S$ with $|U|=k-1$ and $|V|=k$. Since $\bar{w}$ is a permutation of $w$, $w \in \mathbf{W}\left(v_{k, s, t}\right)$ implies $\bar{w} \in \mathbf{W}\left(v_{k, s, t}\right)$, so that

$$
\operatorname{diam}\left(\mathbf{W}\left(v_{k, s, t}\right)\right) \geq\|w-\bar{w}\|_{1}=2 \gamma \cdot\left|S_{1}\right|=2 \gamma \cdot\left\lfloor\frac{s}{2}\right\rfloor \stackrel{s \geq 2}{\geq} \frac{\gamma s}{2} .
$$

If $k \leq \frac{s+1}{2}$ we set $\gamma=\frac{1}{s(2 k-1)} \leq \frac{1}{s}$. For $U, V \subseteq S$ with $|U|=k-1$ and $|V|=k$ we have $w(U) \leq(k-1) \cdot\left(\frac{1}{s}+\gamma\right)$ and $w(V) \geq k \cdot\left(\frac{1}{s}-\gamma\right)$ so that $w(U) \leq w(V)$ and $\operatorname{diam}\left(\mathbf{W}\left(v_{k, s, t}\right)\right) \geq \frac{1}{4 k} \geq \frac{1}{10(s-k)}$.

If $k \geq \frac{s+2}{2}$ we set $\gamma=\frac{1}{s(2 s+3-2 k)} \leq \frac{1}{s}$. For $U, V \subseteq S$ with $|U|=k-1$ and $|V|=k$ we have

and

$$
w(U) \leq \frac{s}{2} \cdot\left(\frac{1}{s}+\gamma\right)+\frac{1}{s}+\left(k-1-\frac{s}{2}-1\right) \cdot\left(\frac{1}{s}-\gamma\right)
$$

so that $w(U) \leq w(V)$ and

$$
w(V) \geq \frac{s}{2} \cdot\left(\frac{1}{s}-\gamma\right)+\frac{1}{s}+\left(k-\frac{s}{2}-1\right) \cdot\left(\frac{1}{s}+\gamma\right)
$$

$$
\operatorname{diam}\left(\mathbf{W}\left(v_{k, s, t}\right)\right) \geq \frac{\gamma s}{2} \geq \frac{1}{2(2(s-k)+3)} \stackrel{s-k \geq 1}{\geq} \frac{1}{10(s-k)} \geq \frac{1}{10 k}
$$

Next we consider the lower bound for $\operatorname{diam}^{\infty}\left(\mathbf{W}\left(v_{k, s, t}\right)\right)$. We set $\gamma=\frac{1}{2 s}, w_{1}=\bar{w}_{2}=$ $\frac{1}{s}+\gamma, w_{2}=\bar{w}_{1}=\frac{1}{s}-\gamma, w_{i}=\bar{w}_{i}=\frac{1}{s}$ for all $3 \leq i \leq s$, and $w_{i}=\bar{w}_{i}=0$ for all $i \in T$. 
It is easily verified that $w \in \mathbb{R}_{>0}^{s+t}$ and $\|w\|_{1}=1$. In order to conclude $w \in \mathbf{W}\left(v_{k, s, t}\right)$ it suffices to check $w(U)+w(T)=w(U) \leq w(V)$ for all $U, V \subseteq S$ with $|U|=k-1$ and $|V|=k$. The latter follows from $w(U) \leq \frac{k-1}{s}+\gamma$ and $w(V) \geq \frac{k}{s}-\gamma$. Since $\bar{w}$ is a permutation of $w$, we also have $\bar{w} \in \mathbf{W}\left(v_{k, s, t}\right)$, so that

$$
\operatorname{diam}^{\infty}\left(\mathbf{W}\left(v_{k, s, t}\right)\right) \geq\|w-\bar{w}\|_{\infty}=2 \gamma=\frac{1}{s} .
$$

For the excluded cases $k=s$ we have:

Lemma 3.2. For integers $s \geq 1$ and $t \geq 0$ with $t+s \geq 2$ we have

$$
\operatorname{diam}\left(\mathbf{W}\left(v_{s, s, t}\right)\right) \geq \frac{2}{3} \quad \text { and } \quad \operatorname{diam}^{\infty}\left(\mathbf{W}\left(v_{s, s, t}\right)\right) \geq \frac{1}{3} .
$$

Proof. Proof. Let $0<\varepsilon<\frac{1}{s}$ be arbitrary. If $s \geq 2$ we choose $w_{1}=\bar{w}_{s}=1-(s-1) \varepsilon$, $w_{i}=\bar{w}_{s+1-i}=\varepsilon$ for all $2 \leq i \leq s$, and $w_{i}=\bar{w}_{i}=0$ for all $s+1 \leq i \leq s+t$. We can easily check $w, \bar{w} \in \mathbf{W}\left(v_{s, s, t}\right)$. Since $\|w-\bar{w}\|_{1}=2 \cdot(1-s \varepsilon)$ and $\|w-\bar{w}\|_{\infty}=1-s \varepsilon$ we have $\operatorname{diam}\left(\mathbf{W}\left(v_{s, s, t}\right)\right) \geq \frac{2}{3}$ and $\operatorname{diam}^{\infty}\left(\mathbf{W}\left(v_{s, s, t}\right)\right) \geq \frac{1}{3}$ using $\varepsilon<\frac{2}{3 s}$.

If $s=1$ then we consider $w=(1,0,0, \ldots, 0) \in \mathbf{W}\left(v_{1,1, t}\right)$ and $\bar{w}=\left(\frac{2}{3}, \frac{1}{3}, 0, \ldots, 0\right) \in$ $\mathbf{W}\left(v_{1,1, t}\right)$. Thus, $\operatorname{diam}\left(\mathbf{W}\left(v_{1,1, t}\right)\right) \geq\|w-\bar{w}\|_{1}=\frac{2}{3}$ and $\operatorname{diam}^{\infty}\left(\mathbf{W}\left(v_{1,1, t}\right)\right) \geq \| w-$ $\bar{w} \|_{\infty}=\frac{1}{3}$.

Next we show that for a given relative quota $q \in(0,1]$ or a given maximum relative weight $\Delta \in(0,1]$ we can construct a weighted game $v$, for any suitably large number of players, with matching representation such that $\operatorname{diam}(\mathrm{W}(v))$ is lower bounded by a positive constant independent of $q$ or $\Delta$. Actually, we construct two representations of the same weighted game and give a lower bound for the distance between the two normalized weight vectors.

Lemma 3.3. For each $q \in(0,1]$ there exists a weighted game $v=[q ; w]=[q ; \bar{w}]$ with $n \geq 2$ players, where $w, \bar{w} \in \mathbb{R}_{\geq 0}^{n}$, and $\|w\|_{1}=\|\bar{w}\|_{1}=1$, such that $\|w-\bar{w}\|_{\infty} \geq \frac{1}{3}$ and $\|w-\bar{w}\|_{1} \geq \frac{2}{3}$.

Proof. Proof. We give general constructions for different ranges of $q$ :

$$
\begin{aligned}
& -\frac{2}{3}<q \leq 1: w=\left(\frac{2}{3}, \frac{1}{3}, 0, \ldots, 0\right), \bar{w}=\left(\frac{1}{3}, \frac{2}{3}, 0, \ldots, 0\right) \\
& \text { - } \frac{1}{3}<q \leq \frac{2}{3}: w=\left(\frac{2}{3}, \frac{1}{3}, 0, \ldots, 0\right), \bar{w}=(1,0, \ldots, 0) \\
& \text { - } 0<q \leq \frac{1}{3}: w=\left(\frac{2}{3}, \frac{1}{3}, 0, \ldots, 0\right), \bar{w}=\left(\frac{1}{3}, \frac{2}{3}, 0, \ldots, 0\right) .
\end{aligned}
$$

Lemma 3.4. Let $\Delta \in(0,1]$ and $n \geq \frac{1}{\Delta}+1$. There exist $w, \bar{w} \in \mathbb{R}_{\geq 0}^{n}, q, \bar{q} \in(0,1]$ with $\|w\|_{1}=\|\bar{w}\|_{1}=1, \Delta(w)=\Delta,[q ; w]=[\bar{q}, \bar{w}]$, and $\frac{1}{2} \cdot\|w-\bar{w}\|_{1} \geq\|w-\bar{w}\|_{\infty} \geq \frac{1}{7}$.

Proof. Proof. We set $s=\left\lfloor\frac{1}{\Delta}\right\rfloor \geq 1$ and $t=n-s \geq 1$, since $n \geq \frac{1}{\Delta}+1 \geq s+1$. For $w=(\Delta, \ldots, \Delta, 1-s \cdot \Delta, 0, \ldots, 0) \in \mathbb{R}_{\geq 0}^{n}$, with $s$ entries being equal to $\Delta$, we have $\Delta(w)=\Delta$ and $[q ; w] \in \mathrm{W}\left(v_{s, s, t}\right)$, where $0<q=s \Delta \leq 1$. Due to Lemma 3.2 we have 
$\operatorname{diam}^{\infty}\left(\mathbf{W}\left(v_{s, s, t}\right)\right) \geq \frac{1}{3}$, so that the triangle inequality implies the existence of a vector $w^{\prime} \in \mathbf{W}\left(v_{s, s, t}\right)$ with $\left\|w-w^{\prime}\right\|_{\infty} \geq \frac{1}{6}$. If $w^{\prime}$ is on the boundary of $\mathbf{W}\left(v_{s, s, t}\right)$ we slightly perturb $w^{\prime}$ to $\bar{w}$ in the interior of $\mathrm{W}\left(v_{s, s, t}\right)$ and complete it to a representation $(\bar{q}, \bar{w})$ with $\bar{q} \in(0,1],[q ; w]=[\bar{q}, \bar{w}]$, and $\|w-\bar{w}\|_{\infty} \geq \frac{1}{7}$. The inequality $\frac{1}{2} \cdot\|w-\bar{w}\|_{1} \geq \mid w-\bar{w} \|_{\infty}$ follows from Lemma 2.3.

By a tailored construction we can obtain a slightly more general result:

Lemma 3.5. For each $\Delta \in(0,1)$ there exists a weighted game $v=[q ; w]=[q ; \bar{w}]$ with $n \geq \frac{4}{3 \Delta}+6$ players, where $q \in(0,1), w, \bar{w} \in \mathbb{R}_{>0}^{n}, \Delta(w)=\Delta(\bar{w})=\Delta$, and $\|w\|_{1}=\|\bar{w}\|_{1}=1$, such that $\|w-\bar{w}\|_{1} \geq \frac{2}{3}$ and $\|w-\bar{w}\|_{\infty} \geq \Delta / 2$.

Proof. Proof. If $\Delta \geq \frac{2}{3}$, we can consider a weighted game with two passers and $n-2$ null players. One representation is given by $q=1-\Delta$ and $w=(\Delta, 1-\Delta, 0, \ldots, 0)$. Of course we can swap the weights of the first two players and obtain a second representation given by quota $q$ an weight vector $\bar{w}=(1-\Delta, \Delta, 0, \ldots, 0)$. With this, we compute $\|w-\bar{w}\|_{1}=2 \cdot(2 \Delta-1) \geq \frac{2}{3}$ and $\|w-\bar{w}\|_{\infty}=2 \Delta-1 \geq \Delta / 2$.

If $0<\Delta<\frac{2}{3}$, we define an integer $a:=\left\lfloor\frac{2}{3 \Delta}\right\rfloor \geq 1$ and consider a weighted game with $2 a$ passers and $n-2 a$ null players. One representation is given by $q=\Delta / 2$, $w_{2 i-1}=\Delta, w_{2 i}=\Delta / 2$ for $1 \leq i \leq a, w_{2 a+1}=w_{2 a+3}=w_{2 a+5}=\frac{1}{3}-\frac{a \Delta}{2} \geq 0$, $w_{2 a+2}=w_{2 a+4}=w_{2 a+6}=0$, and $w_{i}=0$ for all $2 a+7 \leq i \leq n$. By assumption we have $n \geq 2 a+6$ and the first $2 a$ players are obviously passers. By checking $0 \leq$ $\frac{1}{3}-\frac{a \Delta}{2}<\frac{\bar{\Delta}}{2}$ we conclude that the remaining players are null players and have a nonnegative weight. By construction, the weights of the $n$ players sum up to one. Changing the weights of player $2 i-1$ and player $2 i$ for $1 \leq i \leq a$ does not change the game so that we obtain a second representation with quota $q$ and weights $\bar{w}_{2 i}=\Delta, \bar{w}_{2 i-1}=\Delta / 2$ for $1 \leq i \leq a, \bar{w}_{2 a+2}=\bar{w}_{2 a+4}=\bar{w}_{2 a+6}=\frac{1}{3}-\frac{a \Delta}{2} \geq 0, w_{2 a+1}=w_{2 a+3}=w_{2 a+4}=$ $\bar{w}_{2 a+1}=\bar{w}_{2 a+2}=\bar{w}_{2 a+3}=0$, and $\bar{w}_{i}=0$ for all $2 a+7 \leq i \leq n$. With this, we have $\|w-\bar{w}\|_{1}=a \Delta+2-3 a \Delta=2(1-a \Delta) \geq \frac{2}{3}$ and $\|w-\bar{w}\|_{\infty}=\Delta / 2$.

For each $w, \bar{w} \in \mathbb{R}^{n}$ with $\Delta(w)=\Delta(\bar{w})$, we obviously have $\|w-\bar{w}\|_{\infty} \leq \Delta(w)$. So, a constant lower bound for the $\mid \cdot \|_{\infty}$-norm can only exist if we slightly weaken the assumptions as done in Lemma 3.4 .

In some applications only weighted games with a quota of at least one half are considered which clashes with some of our constructions in the proofs of the previous lemmas. However, by considering the dual of a given weighted game we can turn a quota below one half to a quota above one half, see Lemma 2.1. So, instead of small quotas we get large quotas.

So, either knowing the relative quota or the maximum relative weight is not sufficient in order to deduce a non-constant upper bound on the diameter of the weight polytope for a suitably large number of players. However, as we will see in the next section, knowing the relative quota and the maximum relative weight is indeed sufficient for such an upper bound, see Theorem 4.4. Our next aim is to show that this upper bound is tight up to a constant. 
Lemma 3.6. For each $0<q<1,0<\Delta \leq 1$, and each integer $n \geq \frac{1}{\Delta}+2$ there exist weight vectors $w, \bar{w} \in \mathbb{R}_{\geq 0}^{n}$ with $\|w\|_{1}=\|\bar{w}\|_{1}=1, \Delta(w)=\Delta$ and a quota $0<\bar{q} \leq 1$ with $[q ; w]=[\bar{q} ; \bar{w}]$ such that

$$
\|w-\bar{w}\|_{1} \geq \frac{1}{200} \cdot \min \left\{2, \frac{4 \Delta}{\min \{q, 1-q\}}\right\} .
$$

Under the same assumptions there exist weight vectors $w, \bar{w} \in \mathbb{R}_{\geq 0}^{n}$ with $\|w\|_{1}=$ $\|\bar{w}\|_{1}=1, \Delta(w)=\Delta$ and a quota $0<\bar{q} \leq 1$ with $[q ; w]=[\bar{q} ; \bar{w}]$ such that $\|w-\bar{w}\|_{\infty} \geq \frac{\Delta}{5}$.

Proof. Proof. We set $a=\left\lfloor\frac{1}{\Delta}\right\rfloor \geq 1$ and choose the unique integer $b$ with $b \Delta<q$ and $(b+1) \Delta \geq q$. With this we set $k=b+1 \geq 1$ and $w=(\Delta, \ldots, \Delta, 1-a \Delta, 0, \ldots, 0)$, where $0 \leq 1-a \Delta<\Delta$, so that $w \in \mathbb{R}_{>0}^{n}$ and $\|w\|_{1}=1$. If $b \Delta+(1-a \Delta)<q$ we set $s=a$ and $s=a+1$ otherwise, so that $[q ; w]=v_{k, s, n-s}$. Note that $n-s \geq 1$.

If $k=s$, then Lemma 3.2 gives $\operatorname{diam}\left(\mathrm{W}\left(v_{s, s, t}\right)\right) \geq \frac{2}{3}$, so that the triangle inequality implies the existence of a vector $w^{\prime} \in \mathbf{W}\left(v_{s, s, t}\right)$ with $\left\|w-w^{\prime}\right\|_{1} \geq \frac{1}{3}$. If $k<s$, then Lemma 3.1 gives $\operatorname{diam}\left(\mathbf{W}\left(v_{k, s, t}\right)\right) \geq \max \left\{\frac{1}{10 k}, \frac{1}{10(s-k)}\right\}$, so that the triangle inequality implies the existence of a vector $w^{\prime} \in \mathbf{W}\left(v_{k, s, t}\right)$ with

$$
\left\|w-w^{\prime}\right\|_{1} \geq \max \left\{\frac{1}{20 k}, \frac{1}{20(s-k)}\right\}=\frac{1}{20 s} \cdot \frac{1}{\min \left\{\frac{k}{s}, \frac{s-k}{s}\right\}} .
$$

If $k=1$ or $s-k=1$, then $\left\|w-w^{\prime}\right\|_{1} \geq \frac{1}{20}$. In the following we assume $k \geq 2$ and $s-k \geq 2$. By construction we have $\frac{k}{2} \leq(k-1) \Delta<q, k \Delta \geq q$, and $(s-1) \Delta \leq 1$, so that $k<\frac{2 q}{\Delta}, \frac{s-k}{2} \Delta \leq(s-1) \Delta-k \Delta \leq 1-q$ and $s-k \leq \frac{2(1-q)}{\Delta}$.

If $k \leq s-k$ then $q \leq \frac{1}{2}$ and

$$
\left\|w-w^{\prime}\right\|_{1} \geq \frac{1}{20 s} \cdot \frac{1}{\min \left\{\frac{k}{s}, \frac{s-k}{s}\right\}}=\frac{1}{20 k} \geq \frac{1}{40} \cdot \frac{\Delta}{q}=\frac{1}{40} \cdot \frac{\Delta}{\min \{q, 1-q\}} .
$$

If $k>s-k$ then $q>\frac{1}{2}$ and

$$
\left\|w-w^{\prime}\right\|_{1} \geq \frac{1}{20 s} \cdot \frac{1}{\min \left\{\frac{k}{s}, \frac{s-k}{s}\right\}}=\frac{1}{20(s-k)} \geq \frac{1}{40} \cdot \frac{\Delta}{1-q}=\frac{1}{40} \cdot \frac{\Delta}{\min \{q, 1-q\}} .
$$

Thus,

$$
\left\|w-w^{\prime}\right\|_{1} \geq \frac{1}{160} \cdot \min \left\{2, \frac{4 \Delta}{\min \{q, 1-q\}}\right\}
$$

in all cases. If $w^{\prime}$ is on the boundary of $\mathrm{W}\left(v_{k, s, n-s}\right)$, then we slightly perturb $w^{\prime}$ to $\bar{w}$ in the interior of $\mathrm{W}\left(v_{k, s, n-s}\right)$ and choose a quota $\bar{q} \in(0,1]$ such that $[\bar{q} ; \bar{w}]=v_{k, s, n-s}$. This gives the statement for the $\|\cdot\|_{1}$-distance.

For the $\|\cdot\|_{\infty}$-distance we choose $w$ with $[q ; w]=v_{k, s, n-s}$ as above. If $k=s$, then Lemma 3.2 gives $\operatorname{diam}^{\infty}\left(\mathrm{W}\left(v_{s, s, t}\right)\right) \geq \frac{1}{3}$, so that the triangle inequality implies the existence of a vector $w^{\prime} \in \mathrm{W}\left(v_{s, s, t}\right)$ with $\left\|w-w^{\prime}\right\|_{\infty} \geq \frac{1}{6}$. If $k<s$, then Lemma 3.1 
gives $\operatorname{diam}^{\infty}\left(\mathrm{W}\left(v_{k, s, t}\right)\right) \geq \frac{1}{s}$, so that the triangle inequality implies the existence of a vector $w^{\prime} \in \mathbf{W}\left(v_{k, s, t}\right)$ with $\left\|w-w^{\prime}\right\|_{\infty} \geq \frac{1}{2 s}$. For $s=1$ this gives $\left\|w-w^{\prime}\right\|_{\infty} \geq \frac{1}{2}$. For $s \geq 2$ we have $s \leq \frac{2}{\Delta}$ so that $\left\|w-w^{\prime}\right\|_{\infty} \geq \frac{\Delta}{4}$. Since $\Delta \leq 1$ we have $\left\|w-w^{\prime}\right\|_{\infty} \geq \frac{\Delta}{4}$ in all cases, so that the stated result follows possibly by a perturbation.

\section{UPPER BOUNDS FOR THE DIAMETER OF THE WEIGHT POLYTOPE.}

Before we start to upper bound $\operatorname{diam}(\mathrm{W}(v))$ in terms of $\Delta$ and $q$, we provide a slightly more general result.

Lemma 4.1. Let $w \in \mathbb{R}_{\geq 0}^{n}$ with $\|w\|_{1}=1$ for an integer $n \in \mathbb{N}_{>0}$ and $0<q<1$. For each $x \in \mathbb{R}_{\geq 0}^{n}$ with $\|x\|_{1}=1$ and $x(S)=\sum_{s \in S} x_{s} \geq q$ for every winning coalition $S$ of $[q ; w]$, we have

$$
\|w-x\|_{1} \leq \frac{2 \Delta}{\min \{q+\Delta, 1-q\}} \leq \frac{2 \Delta}{\min \{q, 1-q\}},
$$

where $\Delta=\Delta(w)$.

Proof. Proof. Consider a winning coalition $T$ such that $x(T)$ is minimal and invoke $x(T) \geq q$, see the appendix for the technical details.

From Lemma 4.1 we can directly conclude:

Corollary 4.2. Let $w, \bar{w} \in \mathbb{R}_{\geq 0}^{n}$ with $\|w\|_{1}=\|\bar{w}\|_{1}=1$ for an integer $n \in \mathbb{N}_{>0}$ and $0<q, \bar{q}<1$. If $[q ; w]=[\bar{q} ; \bar{w}]$, then we have

$$
\|w-\bar{w}\|_{1} \leq \max \left\{\frac{2 \Delta(w)}{\min \{q, 1-q\}}, \frac{2 \Delta(\bar{w})}{\min \{\bar{q}, 1-\bar{q}\}}\right\} \leq \frac{2 \Delta(w)}{\min \{q, 1-q\}}+\frac{2 \Delta(\bar{w})}{\min \{\bar{q}, 1-\bar{q}\}} .
$$

Unfortunately, this does not allow us to derive a bound on $\|w-\bar{w}\|_{1}$ which only depends on $q$ and $\Delta(w)$. However, we can obtain the following analog of Lemma 4.1 for losing instead of winning coalitions.

Lemma 4.3. Let $w \in \mathbb{R}_{\geq 0}^{n}$ with $\|w\|_{1}=1, \Delta=\Delta(w)$, and $0<q<1$. For each $x \in \mathbb{R}_{\geq 0}^{n}$ with $\|x\|_{1}=1$ and $x(S)=\sum_{s \in S} x_{s} \leq q$ for every losing coalition $S$ of $[q ; w]$, we have

$$
\|w-x\|_{1} \leq \frac{4 \Delta}{\min \{q, 1-q\}} .
$$

Moreover, if $q>\Delta$, then $\|w-x\|_{1} \leq \frac{2 \Delta}{\min \{q-\Delta, 1-q+\Delta\}} \leq \frac{2 \Delta}{\min \{q-\Delta, 1-q\}}$.

Proof. Proof. Consider a losing coalition $T$ such that $x(T)$ is maximal and invoke $x(T) \leq q$. Technical details are provided in the appendix.

Theorem 4.4. Let $w, \bar{w} \in \mathbb{R}_{\geq 0}^{n}$ with $\|w\|_{1}=\|\bar{w}\|_{1}=1, \Delta=\Delta(w)$, and $0<q, \bar{q}<1$. If $[q ; w]=[\bar{q} ; \bar{w}]$, then we have

$$
\|w-\bar{w}\|_{1} \leq \min \left\{2, \frac{4 \Delta}{\min \{q, 1-q\}}\right\} \leq \frac{4 \Delta}{\min \{q, 1-q\}},
$$


i.e., $\operatorname{diam}(\mathrm{W}([q ; w])) \leq \frac{4 \Delta(w)}{\min \{q, 1-q\}}$. Moreover, if $q>\Delta$, then we have

$$
\|w-\bar{w}\|_{1} \leq \frac{2 \Delta}{\min \{q-\Delta, 1-q\}} .
$$

Proof. Proof. In Section 2 we have observed $\|w-\bar{w}\|_{1} \leq 2$. If $\bar{q} \geq q$, then $\bar{w}(S) \geq \bar{q} \geq$ $q$ for every winning coalition $S$ of $[q ; w]$. Here, we can apply Lemma 4.1. Otherwise we have $\bar{w}(T)<\bar{q}<q$ for every losing coalition $T$ of $[q ; w]$ and Lemma 4.3 applies.

\section{Applications.}

A power index $\varphi$ is a mapping from the set of weighted games on $n$ players into $\mathbb{R}_{\geq 0}$. We call $\varphi$ efficient if $\|\varphi(v)\|_{1}=1$ for all weighted games $v$. The difference $\|w-\varphi([q ; w])\|_{1}$ between relative weights and the corresponding power distribution is studied in the literature, see e.g. [5, 10, 15]. Lemma 4.1] is a generalization of [10, Lemma 1]: if $\varphi$ is the nucleolus, see e.g. [17], and $0<q<1$ then

$$
\|w-\varphi([q ; w])\|_{1} \leq \frac{2 \Delta(w)}{\min \{q, 1-q\}}
$$

for all $w \in \mathbb{R}_{\geq 0}^{n}$ with $\|w\|_{1}=1$. From Theorem 4.4 we directly conclude:

Corollary 5.1. Let $w \in \mathbb{R}_{\geq 0}^{n}$ with $\|w\|_{1}=1$ and $0<q<1$. If an efficient power index $\varphi$ permits the existence of a quota $q^{\prime} \in(0,1)$ such that $\left[q^{\prime} ; \varphi([q ; w])\right]=[q ; w]$, i.e., the power vector of the given weighted game can be completed to a representation of the same game, then

$$
\|w-\varphi([q ; w])\|_{1} \leq \frac{4 \Delta(w)}{\min \{q, 1-q\}} .
$$

Representation compatibility of $\varphi$ for $[q ; w]$ is automatically satisfied for the modified nucleolus (modiclus) [19], minimum sum representation index [6] or one of the power indices based on averaged representations [8] for all weighted games and for the Penrose-Banzhaf index for all spherically separable simple games [7]. The theorem also applies to the bargaining model for weighted games analyzed in [16], cf. [14].

For the other direction we have:

Lemma 5.2. Let $n \in \mathbb{N}_{>0}, q, \bar{q} \in(0,1], w, \bar{w} \in \mathbb{R}_{>0}^{n}$ with $\|w\|_{1}=\|\bar{w}\|_{1}=1$ and $[q ; w]=[\bar{q} ; \bar{w}],\|\cdot\|$ be an arbitrary norm on $\mathbb{R}^{n}$ and $\varphi$ be a mapping from the set of weighted games (on $n$ players) into $\mathbb{R}_{\geq 0}^{n}$, then we have

$$
\max \{\|w-\varphi([q ; w])\|,\|\bar{w}-\varphi([\bar{q} ; \bar{w}])\|\} \geq \frac{\|w-\bar{w}\|}{2} .
$$

Proof. Proof. Using the triangle inequality yields $\|w-\varphi([q ; w])\|+\|\bar{w}-\varphi([\bar{q} ; \bar{w}])\| \geq$ $\|w-\bar{w}\|$ from which we can conclude the stated inequality.

Proposition 5.3. Let $\varphi$ be a mapping from the set of weighted games (on n players) into $\mathbb{R}_{\geq 0}^{n}$. 
(i) For each $q \in(0,1]$ and each integer $n \geq 2$ there exists a weighted game $[q ; w]$, where $w \in \mathbb{R}_{\geq 0}^{n}$ and $\|w\|_{1}=1$, such that $\|w-\varphi([q ; w])\|_{1} \geq \frac{1}{3}$ and $\| w-$ $\varphi([q ; w]) \|_{\infty} \geq \frac{1}{6}$.

(ii) For each $\Delta \in(0,1)$ and each integer $n \geq \frac{4}{3 \Delta}+6$ there exists a weighted game $[q ; w]$, where $q \in(0,1], w \in \mathbb{R}_{\geq 0}^{n},\|w\|_{1}=1$, and $\Delta(w)=\Delta$, such that $\|w-\varphi([q ; w])\|_{1} \geq \frac{1}{3}$, and $\|w-\varphi([q ; w])\|_{\infty} \geq \Delta / 4$.

Proof. Proof. Combine Lemma 5.2 with lemmas 3.3 and 3.5

Proposition 5.4. Let $\varphi$ be a mapping from the set of weighted games (on $n$ players) into $\mathbb{R}_{\geq 0}^{n}$. For each $q \in(0,1), \Delta \in(0,1]$, there exist $w, \bar{w} \in \mathbb{R}_{\geq 0}^{n}, \bar{q} \in(0,1]$ with $\|w\|_{1}=\|\bar{w}\|_{1}=1, \Delta(w)=\Delta,[q ; w]=[\bar{q} ; \bar{w}]$, and

$$
\|\bar{w}-\varphi([\bar{q} ; \bar{w}])\|_{1} \geq \frac{1}{200} \cdot \min \left\{2, \frac{4 \Delta}{\min \{q, 1-q\}}\right\} .
$$

Proof. Proof. We construct $w$ as in the proof of Lemma 3.6 and choose integers $k, s$, and $t$ such that $[q ; w]=v_{k, s, t}$. In the proof of Lemma 3.6 we have actually verified

$$
\operatorname{diam}(\mathrm{W}([q ; w])) \geq \frac{1}{80} \cdot \min \left\{2, \frac{4 \Delta}{\min \{q, 1-q\}}\right\}=: \Lambda .
$$

Now choose $w^{\prime}, w^{\prime \prime} \in \mathrm{W}([q ; w])$ with $\left\|w^{\prime}-w^{\prime \prime}\right\|_{1} \geq \Lambda$. By the triangle inequality we have either $\left\|w^{\prime}-\varphi([q ; w])\right\|_{1} \geq \Lambda / 2$ or $\left\|w^{\prime \prime}-\varphi([q ; w])\right\|_{1} \geq \Lambda / 2$. By choosing $\bar{w}$ as $w^{\prime}$ or $w^{\prime \prime}$ and eventually moving it into the interior of $\mathrm{W}([q ; w])$ we obtain the stated result.

\section{CONCLUSion.}

In Theorem 4.4 we have shown that

$$
\operatorname{diam}(\mathrm{W}([q ; w])) \leq \min \left\{2, \frac{4 \Delta}{\min \{q, 1-q\}}\right\} \leq \frac{4 \Delta}{\min \{q, 1-q\}},
$$

for any $q \in(0,1)$ and any $w \in \mathbb{R}_{\geq 0}^{n}$ with $\|w\|_{1}=1$. Lemma 3.6 certifies that this upper bound is in general, i.e., in the worst case, tight up to a constant. (This paper traded smaller constants for easier proofs.) The super-exponential growth of the number of weighted games, see [21] indicates that this is not the case for the majority of weighted games. Thus, it would be interesting to determine other parameters of a representation of a weighted game that permit tight upper bounds on the diameter of the corresponding weight polytope. As shown in Section 5, there are connections to approximations of power indices by weight vectors. Proposition 5.4 gives a partial explanation for the conditions of the main theorem of [15] on a limit result for the Shapley-Shubik index.

\section{APPENDiX A. DELAYED PROOFS}

Proof. Proof of Lemma 2.4. For $n=1$, we have $w_{1}=1, \Delta(w)=1, \alpha=0$, and $L(w)=1$, so that we assume $n \geq 2$ in the remaining part of the proof. For $w_{i} \geq w_{j}$ 
consider $a:=\frac{w_{i}+w_{j}}{2}$ and $x:=w_{i}-a$, so that $w_{i}=a+x$ and $w_{j}=a-x$. With this we have $w_{i}^{2}+w_{j}^{2}=2 a^{2}+2 x^{2}$ and $\left(w_{i}+y\right)^{2}+\left(w_{j}-y\right)^{2}=2 a^{2}+2(x+y)^{2}$. Let us assume that $w^{\star}$ minimizes $\sum_{i=1}^{n} w_{i}^{2}$ under the conditions $w \in \mathbb{R}_{\geq 0},\|w\|_{1}=1$, and $\Delta(w)=\Delta$. (Since the target function is continuous and the feasible set is compact and non-empty, a global minimum indeed exists.) W.l.o.g. we assume $w_{1}^{\star}=\Delta$. If there are indices $2 \leq i, j \leq n$ with $w_{i}^{\star}>w_{j}^{\star}$, i.e., $x>0$ in the above parameterization, then we may choose $y=-x$. Setting $w_{i}^{\prime}:=w_{i}^{\star}+y=a=\frac{w_{i}^{\star}+w_{j}^{\star}}{2}, w_{j}^{\prime}:=w_{j}^{\star}-y=a=\frac{w_{i}^{\star}+w_{j}^{\star}}{2}$, and $w_{h}^{\prime}:=w_{h}^{\star}$ for all $1 \leq h \leq n$ with $h \notin\{i, j\}$, we have $w^{\prime} \in \mathbb{R}_{\geq 0}^{n},\left\|w^{\prime}\right\|_{1}=1, \Delta\left(w^{\prime}\right)=\Delta$, and $\sum_{h=1}^{n}\left(w_{h}^{\prime}\right)^{2}=\sum_{h=1}^{n}\left(w_{h}^{\star}\right)^{2}-x^{2}$. Since this contradicts the minimality of $w^{\star}$, we have $w_{i}^{\star}=w_{j}^{\star}$ for all $2 \leq i, j \leq n$, so that we conclude $w_{i}^{\star}=\frac{1-\Delta}{n-1}$ for all $2 \leq i \leq n$ from $1=\left\|w^{\star}\right\|_{1}=\sum_{h=1}^{n} w_{h}^{\star}$. Thus, $L(w) \leq 1 /\left(\Delta^{2}+\frac{(1-\Delta)^{2}}{n-1}\right)$, which is tight. Since $\Delta \leq 1$ and $n \geq 2$, we have $1 /\left(\Delta^{2}+\frac{(1-\Delta)^{2}}{n-1}\right) \leq \frac{1}{\Delta^{2}}$, which is tight if and only if $\Delta=1$, i.e., $n-1$ of the weights have to be equal to zero.

Now, let us assume that $w$ maximizes $\sum_{i=1}^{n} w_{i}^{2}$ under the conditions $w \in \mathbb{R}_{\geq 0}$, $\|w\|_{1}=1$, and $\Delta(w)=\Delta$. (Due to the same reason a global maximum indeed exists.) Due to $1=\|w\|_{1} \leq n \Delta$ we have $0<\Delta \leq 1 / n$, where $\Delta=1 / n$ implies $w_{i}=\Delta$ for all $1 \leq i \leq n$. In that case we have $L(w)=n$ and $\alpha=0$, so that the stated lower bounds for $L(w)$ are valid. In the remaining cases we assume $\Delta>1 / n$. If there would exist two indices $1 \leq i, j \leq n$ with $w_{i} \geq w_{j}, w_{i}<\Delta$, and $w_{j}>0$, we may strictly increase the target function by moving weight from $w_{j}$ to $w_{i}$ (this corresponds to choosing $y>0$ ), by an amount small enough to still satisfy the constraints $w_{i} \leq \Delta$ and $w_{j} \geq 0$. Since $\Delta>0$, we can set $a:=\lfloor 1 / \Delta\rfloor \geq 0$ with $a \leq n-1$ due to $\Delta>1 / n$. Thus, for a maximum solution, we have exactly $a$ weights that are equal to $\Delta$, one weight that is equal to $1-a \Delta \geq 0$ (which may indeed be equal to zero), and $n-a-1$ weights that are equal to zero. With this and $a \Delta=1-\alpha \Delta$ we have $\sum_{i=1}^{n} w_{i}^{2}=a \Delta^{2}(1-a \Delta)^{2}=$ $\Delta-\alpha \Delta^{2}+\alpha^{2} \Delta^{2}=\Delta\left(1-\alpha \Delta+\alpha^{2} \Delta\right)=\Delta(1-\alpha(1-\alpha) \Delta) \leq \Delta$. Here, the latter inequality is tight if and only if $\alpha=0$, i.e., $1 / \Delta \in \mathbb{N}$.

Proof. Proof of Lemma 4.1. We set $N=\{1, \ldots, n\}, w(U)=\sum_{u \in U} w_{u}$ and $x(U)=$ $\sum_{u \in U} x_{u}$ for each $U \subseteq N$. Let $S^{+}=\left\{i \in N \mid x_{i}>w_{i}\right\}$ and $S^{-}=\left\{i \in N \mid x_{i} \leq w_{i}\right\}$, i.e., $S^{+}$and $S^{-}$partition the set $N$ of players. We have $w\left(S^{+}\right)<1$ since $w\left(S^{+}\right)<$ $x\left(S^{+}\right) \leq x(N)=1$, so that $w\left(S^{-}\right)>0$. Define $0 \leq \delta \leq 1$ by $x\left(S^{-}\right)=(1-\delta) w\left(S^{-}\right)$. We have

$$
x\left(S^{+}\right)=1-x\left(S^{-}\right)=w\left(S^{+}\right)+w\left(S^{-}\right)-(1-\delta) w\left(S^{-}\right)=w\left(S^{+}\right)+\delta w\left(S^{-}\right)
$$

and

$$
\|w-x\|_{1}=\left(x\left(S^{+}\right)-w\left(S^{+}\right)\right)+\left(w\left(S^{-}\right)-x\left(S^{-}\right)\right)=2 \delta w\left(S^{-}\right) .
$$

Generate a set $T$ by starting at $T=\emptyset$ and successively add a remaining player $i$ in $N \backslash T$ with minimal $x_{i} / w_{i}$, where all players $j$ with $w_{j}=0$ are the worst ones. Stop if 
$w(T) \geq q$. By construction $T$ is a winning coalition of $[q ; w]$ with $w(T)<q+\Delta$, since the generating process did not stop earlier and $w_{j} \leq \Delta(w)$ for all $j \in N$.

If $w\left(S^{-}\right) \geq q$, we have $T \subseteq S^{-}$and $x(T) / w(T) \leq x\left(S^{-}\right) / w\left(S^{-}\right)=1-\delta$. Multiplying by $w(T)$ and using $w(T)<q+\Delta$ yields

$$
x(T) \leq(1-\delta) w(T)<(1-\delta)(q+\Delta)=(1-\delta) q+(1-\delta) \Delta .
$$

Since $x(T) \geq q$, as $T$ is a winning coalition, we conclude $\delta<\Delta /(q+\Delta)$. Using this and $w\left(S^{-}\right)<1$ in Equation (2) yields

$$
\|w-x\|_{1}<\frac{2 \Delta}{q+\Delta}<\frac{2 \Delta}{q} .
$$

If $w\left(S^{-}\right)<q$, we have $S^{-} \subseteq T, x(T)=x\left(S^{-}\right)+x\left(T \backslash S^{-}\right), w\left(T \backslash S^{-}\right)>0$, and $w\left(S^{+}\right)>0$. Since $T \backslash S^{-} \subseteq S^{+}, x\left(T \backslash S^{-}\right) / w\left(T \backslash S^{-}\right) \leq x\left(S^{+}\right) / w\left(S^{+}\right)$, so that

$$
\begin{aligned}
x(T) & =x\left(S^{-}\right)+x\left(T \backslash S^{-}\right) \leq(1-\delta) w\left(S^{-}\right)+\frac{x\left(S^{+}\right)}{w\left(S^{+}\right)} \cdot\left(w(T)-w\left(S^{-}\right)\right) \\
& \leq(1-\delta) w\left(S^{-}\right)+\frac{x\left(S^{+}\right)}{w\left(S^{+}\right)} \cdot\left(q+\Delta-w\left(S^{-}\right)\right) \\
& =q+\frac{x\left(S^{+}\right) \Delta-(1-q) \delta w\left(S^{-}\right)}{w\left(S^{+}\right)} \\
& \leq q+\frac{\Delta-(1-q) \delta w\left(S^{-}\right)}{w\left(S^{+}\right)} .
\end{aligned}
$$

Since $x(T) \geq q$, we conclude $(1-q) \delta w\left(S^{-}\right) \leq \Delta$, so that $\|w-x\|_{1} \leq \frac{2 \Delta}{1-q}$.

Proof. Proof of Lemma 4.3. If $q \leq 2 \Delta$, then $\frac{4 \Delta}{\min \{q, 1-q\}} \geq \frac{4 \Delta}{q} \geq 2 \geq\|x-w\|_{1}$, so that we can assume $q>\Delta$.

Using the notation from the proof of Lemma 4.1, we have $x\left(S^{+}\right)=w\left(S^{+}\right)+\delta w\left(S^{-}\right)$ and $\|w-x\|_{1}=2 \delta w\left(S^{-}\right)$.

Generate $T$ by starting at $T=\emptyset$ and successively add a remaining player $i$ in $N \backslash T$ with maximal $x_{i} / w_{i}$, where all players $j$ with $w_{j}=0$ are taken in the first rounds, as long as $w(T)+w_{i}<q$. By construction $T$ is a losing coalition of $[q ; w]$ with $q-\Delta \leq w(T)<q$, since the generating process did not stop earlier.

If $w\left(S^{+}\right) \geq q$, we have $T \subseteq S^{+}$and $x(T) / w(T) \geq x\left(S^{+}\right) / w\left(S^{+}\right)=1+\frac{\delta w\left(S^{-}\right)}{w\left(S^{+}\right)} \geq$ $1+\delta w\left(S^{-}\right)$. Multiplying by $w(T)$ and using $w(T) \geq q-\Delta$ yields $x(T) \geq\left(1+\delta w\left(S^{-}\right)\right) w(T) \geq\left(1+\delta w\left(S^{-}\right)\right)(q-\Delta)=(q-\Delta)+\delta w\left(S^{-}\right)(q-\Delta)$. Since $x(T) \leq q$, as $T$ is a losing coalition, we conclude $\delta w\left(S^{-}\right) \leq \Delta /(q-\Delta)$, so that $\|w-x\|_{1}<\frac{2 \Delta}{q-\Delta}$. 
If $w\left(S^{+}\right)<q$, we have $S^{+} \subseteq T, x(T)=x\left(S^{+}\right)+x\left(T \backslash S^{+}\right), w\left(T \backslash S^{+}\right)>0$, and $w\left(S^{-}\right)>0$. Since $T \backslash S^{+} \subseteq S^{-}, x\left(T \backslash S^{+}\right) / w\left(T \backslash S^{+}\right) \geq x\left(S^{-}\right) / w\left(S^{-}\right)$, so that

$$
\begin{aligned}
x(T) & =x\left(S^{+}\right)+x\left(T \backslash S^{+}\right) \geq w\left(S^{+}\right)+\delta w\left(S^{-}\right)+\frac{x\left(S^{-}\right)}{w\left(S^{-}\right)} \cdot\left(w(T)-w\left(S^{+}\right)\right) \\
& \geq w\left(S^{+}\right)+\delta w\left(S^{-}\right)+(1-\delta) \cdot\left(q-\Delta-w\left(S^{+}\right)\right) \\
& =\delta w\left(S^{-}\right)+q-\Delta-\delta q+\delta \Delta+\delta w\left(S^{+}\right)=q-\Delta+\delta(1-q+\Delta) .
\end{aligned}
$$

Since $x(T) \leq q, \delta \leq \frac{\Delta}{1-q+\Delta}$, so that $\|w-x\|_{1} \leq \frac{2 \Delta}{1-q+\Delta}$ due to $w\left(S^{-}\right) \leq 1$.

So, for $q>\Delta$ we have $\|w-x\|_{1} \leq \frac{2 \Delta}{\min \{q-\Delta, 1-q+\Delta\}} \leq \frac{2 \Delta}{\min \{q-\Delta, 1-q\}}$. In order to show $\|w-x\|_{1} \leq \frac{4 \Delta}{\min \{q, 1-q\}}$ it remains to consider the case $q \leq 1-q$. For $q>2 \Delta$, see the start of the proof, we have $\|w-x\|_{1} \leq \frac{2 \Delta}{\min \{q-\Delta, 1-q\}} \leq \frac{2 \Delta}{q-\Delta} \leq \frac{4 \Delta}{q} \leq \frac{4 \Delta}{\min \{q, 1-q\}}$

\section{REFERENCES}

[1] L. Babai, K. A. Hansen, V. V. Podolskii, and X. Sun. Weights of exact threshold functions. In International Symposium on Mathematical Foundations of Computer Science, pages 66-77. Springer, 2010.

[2] J. F. Banzhaf III. Weighted voting doesn't work: a mathematical analysis. Rutgers Law Review, 19:317, 1964.

[3] G. Chalkiadakis, E. Elkind, and M. Wooldridge. Computational aspects of cooperative game theory. Synthesis Lectures on Artificial Intelligence and Machine Learning, 5(6):1-168, 2011.

[4] B. Curry and K. D. George. Industrial concentration: a survey. The Journal of Industrial Economics, 31(3):203-255, 1983.

[5] P. Dubey and L. S. Shapley. Mathematical properties of the Banzhaf power index. Mathematics of Operations Research, 4(2):99-131, 1979.

[6] J. Freixas and S. Kaniovski. The minimum sum representation as an index of voting power. European Journal of Operational Research, 233(3):739-748, 2014.

[7] N. Houy and W. S. Zwicker. The geometry of voting power: weighted voting and hyper-ellipsoids. Games and Economic Behavior, 84:7-16, 2014.

[8] S. Kaniovski and S. Kurz. The average representation -a cornucopia of power indices? Homo Oeconomicus, 32(2):169-181, 2015.

[9] S. Kaniovski and S. Kurz. Representation-compatible power indices. Annals of Operations Research, pages 1-31, to appear.

[10] S. Kurz, S. Napel, and A. Nohn. The nucleolus of large majority games. Economics Letters, 123(2):139-143, 2014.

[11] M. Laakso and R. Taagapera. Effective number of parties: A measure with application to Western Europe. Comparative Political Studies, 12(1):3-27, 1979.

[12] M. Laakso and R. Taagepera. Proportional representation and effective number of parties in Finland. In Power, voting, and voting power, pages 107-120. Springer, 1982.

[13] D. Leech. Ownership concentration and the theory of the firm: a simple-game-theoretic approach. The Journal of Industrial Economics, pages 225-240, 1987.

[14] M. Montero. Proportional payoffs in legislative bargaining with weighted voting: a characterization. Quarterly Journal of Political Science, 12(3):325-346, 2017.

[15] A. Neyman. Renewal theory for sampling without replacement. The Annals of Probability, pages 464-481, 1982. 
[16] G. Owen, I. Lindner, S. L. Feld, B. Grofman, and L. Ray. A simple "market value" bargaining model for weighted voting games: characterization and limit theorems. International Journal of Game Theory, 35(1):111-128, 2006.

[17] D. Schmeidler. The nucleolus of a characteristic function game. SIAM Journal on Applied Mathematics, 17(6):1163-1170, 1969.

[18] L. S. Shapley and M. Shubik. A method for evaluating the distribution of power in a committee system. American Political Science Review, 48(3):787-792, 1954.

[19] P. Sudhölter. The modified nucleolus as canonical representation of weighted majority games. Mathematics of Operations Research, 21(3):734-756, 1996.

[20] J. von Von Neumann and O. Morgenstern. Theory of Games and Economic Behavior. Princeton University Press, 3rd edition, 1953.

[21] Y. A. Zuev. Asymptotics of the logarithm of the number of threshold functions of the algebra of logic. Soviet Mathematics - Doklady, 39(3):512-513, 1989.

SASCha Kurz, University of Bayreuth, 95440 Bayreuth, Germany

E-mail address: sascha.kurz@uni-bayreuth.de 